\title{
An improved sharpening algorithm for foggy picture based on dark-channel prior
}

\author{
Liu Hui ${ }^{1,2, a}$, He Peng ${ }^{1, b}$ \\ ${ }^{1}$ Research Centre for Application of New Communication Technologies, \\ Chongqing University of Posts and Telecommunications Chongqing (CQUPT), 400065, China \\ ${ }^{2}$ Chongqing Information Technology Designing Co. , LTD Chongqing, 400065, China \\ Chongqing University of Posts and Telecommunications Chongqing (CQUPT), 400065, China \\ aliuhui@cqyit.com, bhe_1024@126.com.
}

Keywords: Image defogging; atmosphere scattering model; Dark-channel prior; Joint bilateral filter

\begin{abstract}
In order to improve the clarity of degraded image in foggy weather, an improved image defogging method based on dark-channel prior is proposed. The original algorithm is sensitive to bright region and needs more computation time. An improved algorithm is proposed based on the work of previous researchers for defogging. Firstly, the sky area is adaptively selected to compute the Atmospheric light intensity. Then, fast bilateral filtering algorithm is used to refine the transmission map which greatly reduces the complexity of the algorithm. Since the color of image after haze removal is lower than the real scene, we propose a simple and effective method to adjust brightness. Experimental results indicate that the algorithm can effectively eliminate the impact of gray and bright areas on the calculations of atmospheric light and transmittance to recover the true color and clarity of the scene, while the time complexity of the algorithm is only a linear function of the input image size that can improve computing speed obviously.
\end{abstract}

\section{Introduction}

In video surveillance, target tracking, self-driving vehicles and other applications, are related to the image processing technology. The performance of these outdoor images processing system is vulnerable to bad weather. In fog, haze and other weather conditions, the reflected light will be the object of absorption and scattering of fine particles in the atmosphere, resulting in a serious decline in image quality, it not only affects the image visual effects, but also seriously interfere with subsequent graphics processing results. Therefore, the research of the image defogging algorithm has practical significance.

In recent years, research has made great progress based on a single image defogging. Tan ${ }^{[1]}$ removes haze according image enhancement based on the methods of maximizing the local contrast of the restored image color contrast, his method achieves good results in certain scenes images, but it is not consistent with the actual physical model that results obtained easily distorted. Fattal ${ }^{[3]}$ assumes the scene shadows and media transmission is not relevant, using independent principal component analysis (ICA) model to estimate the transmission rate, but his algorithm can only handle color image, and it is not ideal for the area of heavy fog. $\mathrm{He}^{[5]}$ proposes dark colors priori rule (statistical results show that most of the outdoor images meet the rule), prior to the use of the transmittance of the strike, and the use of soft matting algorithm to correct transmittance ,we finally recover clear images. The method can get good results for most outdoor pictures, but is not an ideal method to defog as the higher the algorithm complexity.

In this paper, the current poor image restoration techniques to fog effects and time-consuming operations such as lack of dark colors priori method to improve the fog, dark colors, based on the proposed adaptive algorithm sky area, in order to overcome the white object effects of atmospheric light intensity calculated on; matting algorithm based on medium repair transmittance distribution method is proposed to improve the use of bilateral filtering method quick fix, and restore the image colors dim brightness adjustment issues, enabling rapid recovery of high quality images. 


\section{Dark-channel prior defogging algorithm}

\section{Imaging atmospheric scattering model of equation}

In the field of computer vision, usually following physical model to describe the atmospheric scattering model monochrome fog, haze and other inclement weather conditions, the model includes direct attenuation model and airlight model, and its expression of the formula:

$$
I(x)=J(x) e^{-r d(x)}+A\left(1-e^{-r d(x)}\right)
$$

Where $\mathrm{x}$ is image pixel coordinates, $I(x)$ is observed foggy image; $J(x)$ is to be restored haze-free image; $r$ is the atmospheric particle scattering coefficient; $d(x)$ is the scene depth; $A$ is global atmospheric light; $J(x) e^{-r d(x)}$ describes the reflective surface due to the scattering of light propagation effect which is attenuated in the medium; $A\left(1-e^{-r d(x)}\right)$ describe the image color and brightness offset. We use $t(x)$ to replace $e^{-r d(x)}$, then (1) can be simplified as:

$$
I(x)=J(x) t(x)+A(1-t(x))
$$

The purpose of the image is to go through $I(x)$ the fog was already determined $J(x)$, but the formula (2), $t(x)$ and $A$ are unknown, $J(x)$ solving equations become an unrestricted morbid solving problems.

\section{Prior dark colors}

Dark colors priori (dark channel prior) is a single image defogging simple and effective method. The a priori is a large number of statistical regularities outdoor image without fog, that for the vast majority of each local region of the image without fog (not including part of the sky) are present at least some of the low intensity value of a pixel color channel these dots called "dark pixels".

Thus, for dark colors meet outdoor priori no fog image $J(x)$, assuming a local region $\Omega(x)$, then the dark colors can be expressed as:

$$
J^{\text {dark }}(x)=\min _{y \in \Omega(x)}\left(\min _{c \in(r, g, b)}\left(J^{c}(y)\right)\right) \rightarrow 0
$$

Where, $J(y)$ on behalf of the two-dimensional spatial coordinates in the pixel y brightness values for a particular color channel at y.

Preliminary estimates transmittance

He's algorithm utilizing a priori dark colors and atmospheric scattering model can quickly estimate the fog density and transmission imaging time. Assuming transmittance remain unchanged within the local area $\Omega(x)$, equation (2) about the minimum filtering operation performed, can be obtained:

$$
\min _{y \in \Omega(x)}\left(\min _{c \in(r, g, b)}\left(\frac{I^{c}(y)}{A^{c}}\right)\right)=t(x) \min _{y \in \Omega(x)}\left(\min _{c \in(r, g, b)}\left(\frac{J^{c}(y)}{A^{c}}\right)\right)+(1-t(x))
$$

According to the value of the dark colors of the image without fog tends to 0 this a priori, then:

$$
\tilde{t}(x)=1-\min _{y \in \Omega(x)}\left(\min _{c \in(r, g, b)}\left(\frac{I^{c}(y)}{A^{c}}\right)\right)
$$

According to preliminary estimates space perspective principle, in order to maintain the depth information of the image, the introduction of a constant $\omega^{\omega}$ in the formula, targeted to retain part of the fog covering the distant scene, the transmittance can be obtained:

$$
\tilde{t}(x)=1-\omega \min _{y \in \Omega(x)}\left(\min _{c}\left(\frac{I^{c}(y)}{A^{c}}\right)\right)
$$

Where $\omega$ is a variable parameter, with the depth of field and set its value, the value is 0.95 in this article.

\section{Our improved algorithm}

In this paper, based on a priori dark colors, a fast single image to fog algorithms, this algorithm can be divided into three steps:1)calculation of the input image is a dark color, and the brightness of the sky and the initial estimate transmission distribution;2) use of joint bilateral filtering transmittance quick fix; 3)to adjust the brightness on the recovery operation, enhanced image 
contrast, improve visual effects.

\section{Light intensity of the global atmosphere}

He's algorithm foggy image obtaining dark colors, $0.1 \%$ dark channel selected before the pixel value is larger the dark region of the set of primary colors, then in a region corresponding to the original image to traverse to find the maximum luminance pixels, i.e. pixel values corresponding the value of the ambient light. However, in order to filter out the white areas in the image(Fig.1 (b)and 1(d) the that shown in the red box) on sky brightness solving, structure in seeking a dark primary color filter used in the process should be sized larger than the size of the white object, however, if the image size is smaller than the area of the sky filter size, the filter will filter out the process in the wrong area of the sky. Figure 1 (c), the small area of the sky, only to see the sky from the area between the branches, the larger the size of the filter is easy to Sky region completely corrosion.

Because of the relatively large area of the sky luminance and the gray value changes relatively flat. Therefore, we use Canny operator on input image edge detection of gray component of the obtained statistical edge grayscale divided into blocks, Were calculated for each image block in the proportion of edge pixels, denoted by $P_{\text {edge }}(x), I^{\text {dark }}(x)>T_{l}$ and $P_{\text {edge }}(x)<T_{p}$ will meet the set area is designated as a candidate sky region, assuming $95 \%$ of the primary colors $I^{\text {dark }}(x)$ in the dark luminance threshold $T_{l}$ maximum value herein, the threshold $T_{p}$ is 0.001 smoothing. In Fig.1(d) in the candidate region identified by the black sky.

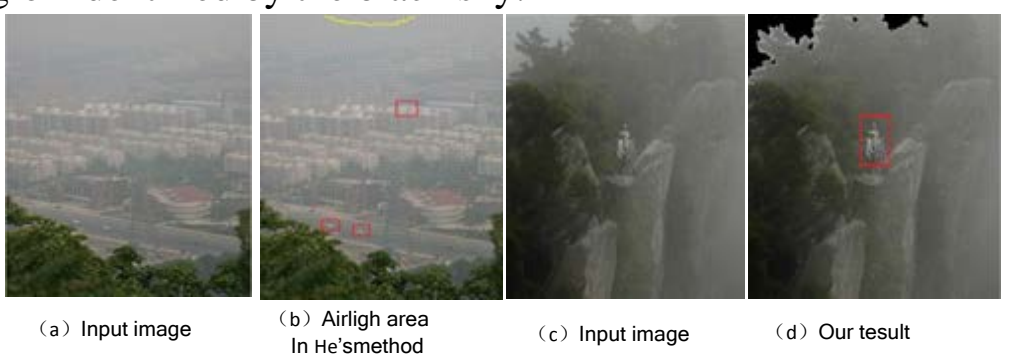

Fig.1 Solving icons of candidate sky region

\section{Repairing transmittance algorithm based on fast bilateral filtering}

Bilateral filtering is a non-iterative method for edge preserving smoothing filter in image processing additive noise can play a role in the advantage. The product of its weight by airspace (Spatial domain) and range (Range domain) smoothing function obtained, and right neighboring pixels a weight with increasing distance from the center pixel and a gradation difference decreases. In this paper, Gaussian bilateral filtering is used when spatial domain and range are Gaussian smoothing function, a priori laws for the use of dark colors obtained transmittance rough estimate using bilateral filtering refinement operation:

$$
t(x)=\frac{1}{W(x)} \sum_{y \in \Omega(x)} G_{\sigma_{s}}(\|x-y\|) G_{\sigma_{r}}(|E(x)-E(y)|) t(y)
$$

Where, $\mathrm{G}$ is Gaussian function, $\sigma_{s}$ control of the spatial domain weights, $\sigma_{r}$ weight control range.Range weights are based on the original brightness image $E(x)$ to calculate the luminance graph, which ensures strong edge information at the original image to be used. $W(x)$ is the sum weights for the center pixel $x$ of the local patch:

$$
W(x)=\sum_{y \in \Omega(x)} G_{\sigma_{s}}(\| x-y \mid) G_{\sigma_{r}}(|E(x)-E(y)|)
$$

\section{1) Fast joint bilateral filtering algorithm}

Bilateral filtering is a nonlinear filtering, fast convolution algorithm traditional airspace is no longer applicable, and according to (6), the direct computation time and space overhead bilateral filtering large, so many scholars are working on methods to accelerate bilateral filtering ${ }^{[8.9]}$. Where Paris $^{[9]}$ will be the basis of bilateral filtering signal processing theory is represented as high-dimensional space of linear time-invariant convolution, the first of the next sampling data, and then perform a low-pass filter in the high-dimensional space, and finally by linear interpolation 
operation get the initial resolution, to get the final filtering results.

In formula (7), the introduction of the variable $\zeta$ represents the gray value of the sampling area $\Omega$,while lead Kronecker symbols $\delta(\xi) . \zeta$ is in the range of the input image on the luminance value range $E(x)$ The weighted average of the bilateral filter becomes homogeneous form as follow:

$$
\left(\begin{array}{l}
W(x) t(x) \\
W(x)
\end{array}\right)=\sum_{y \in \Omega_{x}} \sum_{\zeta \in R} G_{\sigma_{s}}\left(\|x-y\| G_{\sigma_{r}}(E(x)-\zeta \mid) \cdot \delta(\zeta-E(y))(\underset{W(y)}{W(\tilde{C}) t(y)})\right.
$$

\section{2) $\mathbf{T}(\mathrm{x})$ rapidly filtering}

In order to eliminate the edges of the joint bilateral filtering and blurring effects, we use a joint three-dimensional histogram calculation joint bilateral filtering, based on the joint histogram information can retain the characteristics of gray area, thereby increasing the final calculation of the transmission map obtained by interpolation accuracy specifically to achieve the following:

(1)Sampling: set $\tilde{t(x)}$ as an input image, the spatial resolution and the resolution of $\tilde{t(x)}$ amplitude and respectively performs down-sampling $\alpha$ and $\beta$ times, get a three-dimensional space $\left(x_{d}, y_{d}, I_{d}\right)$, where, $x_{d}, y_{d}, I_{d}$ respectively, the horizontal, vertical coordinates and gray values sampled.

(2)Calculation joint histogram: traversing each pixel of the image $\tilde{t(x)}$ to generate three-dimensional array $I\left(x_{d}, y_{d}, I_{d}\right)$, the corresponding value of the accumulation, then $I\left(x_{d}, y_{d}, I_{d}\right)=I\left(x_{d}, y_{d}, I_{d}\right)+E(x)$.

(3)Filtering: we use three-dimensional Gaussian filter on $I\left(x_{d}, y_{d}, I_{d}\right)$ to obtain $I_{G}\left(x_{d}, y_{d}, I_{d}\right)$.

(4)Interpolation: by three-dimensional operation to $I_{G}\left(x_{d}, y_{d}, I_{d}\right)$ which we get above and put linear interpolation operation the next, then we get $t(x)$.

\section{3) Image Restoration}

For the fog area, in order to avoid the process of seeking transmittance noise is amplified, the transmittance set lower limit value $t_{0}$ according to the experimental conditions selected between 0.15 and 0.07, Incremental factor $\Delta(\mathrm{x})$ as transmittance partial superposition, to avoid its transmittance is too small to cause a partial color distortion restoration figure .The resulting image restoration formula:

$$
J(x)=\frac{I(x)-A}{\max \left((t(x)+\Delta(x)), t_{0}\right)}+A
$$

\section{4) Brightness adjustment after defogging image}

In foggy conditions, as a result of atmospheric light fog overall image partial gray color pixel values higher than the actual situation, and therefore recovery plan after the fog color to dim, as shown in Fig.2 (b) shows, therefore need to restore the image brightness adjustment operation, we use the standard deviation truncation method ${ }^{[2]}$. First, set the color saturation threshold $s \in(0,1)$. In the RGB 3 color channels separately calculate the maximum points median $f_{\max }$ and minimum $f_{\text {min }}$, then set range for each color channel as $\left[r_{\text {min }}, r_{\max }\right]$. The brightness of the image is enhanced as follows:

$$
R(x)=\frac{\left(J(x)-f_{\min }\right) \bullet\left(r_{\max }-r_{\min }\right)}{f_{\max }-f_{\min }}+r_{\min }
$$

\section{Comparison and analysis of the experimental results}

We use VS2010 and OpenCV2.4 achieve in this algorithm, the program operating environment for Windows 7, configure the computer to Pentium Dual-Core CPU E5200@2.50 GHZ with 2 GB RAM .Fig. 2 shows the algorithm for each intermediate foggy image processing results, where Fig. 2 (c) $~ 3$ (f) show bilateral filtering on patch based on the calculated transmittance smoothing effect, the results can be seen that the block is well removed, while maintaining the overall outline of the scene. 


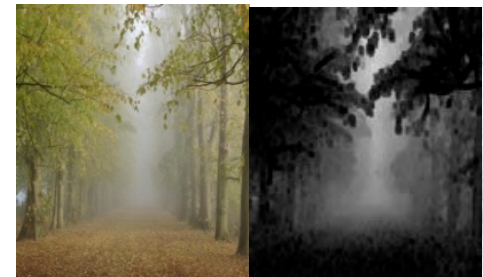

(a)

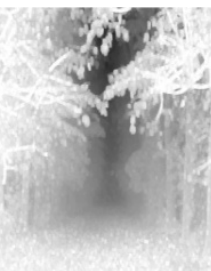

(c)

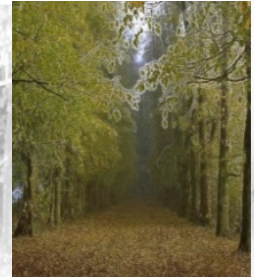

(d)

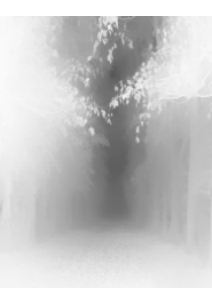

(e)

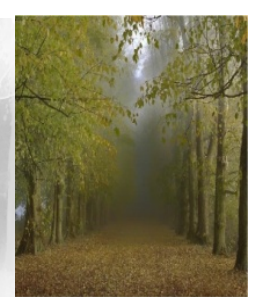

(f)

Fig.2 In-between processed results. (a)input haze image. (b)dark channel. (c)estimated initial transmission map. (d) recovery result with unrefined transmission. (e)refined transmission map after bilateral filter. (f)final haze-free image.

\section{Subjective evaluation}

This algorithm is applied to a large number of outdoor haze have made a better image to the fog effect, Figure 3 shows a comparison of the results of the experimental part of the outdoor scene. As can be seen, the proposed algorithm can effectively solve the interference problem white areas, and better treatment effect in detail and fog areas, better true representation of the actual color of the scene.

\section{Objective evaluation}

Currently, blind evaluation methods is mainly used in the field of image defogging side gradient method visible from Hautiere et $\mathrm{al}^{[6]}$. The method uses the recovery after the new image of the visible side $e$ and the average gradient $\bar{r}$ to evaluation defogging effect:

$$
e=\frac{n_{r}-n_{0}}{n_{0}}, \bar{r}=\frac{\overline{g_{r}}}{\overline{g_{0}}}
$$

Where, $n_{0}$ and $n_{r}$ represent the number of the original image $I(x)$ and the visible image $J(x)$ edges, the number of visible edges contrast to be obtained by calculation; $\overline{g_{r}}$ represent the average gradient of fog image, $\overline{g_{0}}$ represents the average gradient of the original image, the image sharpness average gradient characterization to reflect the richness of image detail. For Scene and Sweden, the results are shown in Tab.1 below.

\begin{tabular}{llrcl}
\multicolumn{4}{c}{ Tab.1 Comparision with He s and Tarel's work } \\
\hline name & Algorithm & Time /s & e & $\bar{r}$ \\
\hline Scene & He & 1.348 & 0.1072 & 1.2731 \\
$366 \times 472$ & Tarel & 2.591 & 0.3951 & 1.3142 \\
& This paper & 0.502 & 0.4278 & 1.3522 \\
\multirow{2}{*}{ Sweden } & He & 18.135 & 0.6055 & 1.4673 \\
$600 \times 400$ & Tare & 3.100 & 0.5391 & 2.0157 \\
& This paper & 1.733 & 0.6992 & 1.5998 \\
\hline
\end{tabular}

As can be seen from the results, in the same image size, the proposed algorithm have improved significantly processing speed with respect to Tarel's and He's algorithm; while adding visible edges than the number of He's. In terms of average gradient, our algorithm has equal result with Tarel's and He's algorithm, basically meets the human visual image detail requirements. Comparative results show that this improved algorithm has certain advantages in terms of defogging.

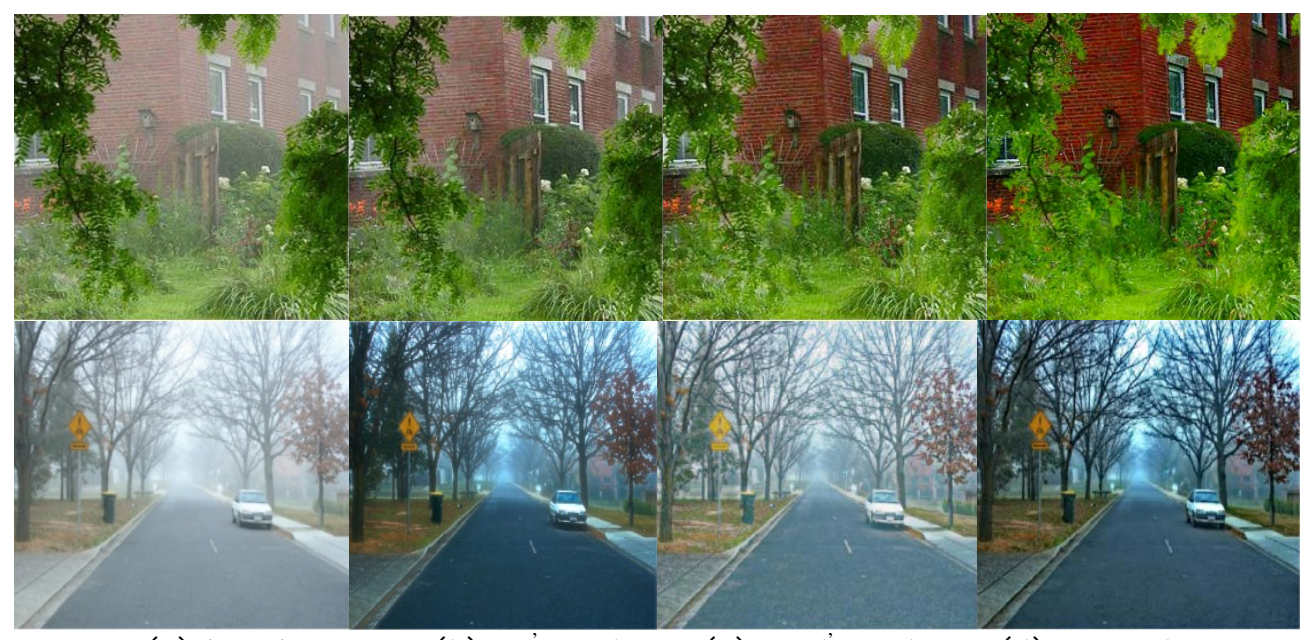
(a) input image
(b) He's results
(c) Tarel's results
(d) our results

Fig.3 Comparision of haze removal results (From top to bottom, respectively Scene and Sweden) 


\section{Summary}

In this paper, the dark colors on the basis of a priori propose an improved algorithm to fog. He's algorithm is poor when the picture when there is a large area of white fog effects to regional interference and algorithms for time-consuming problems were repaired to improve both the strike and the transmittance of the atmosphere from the light intensity values. Through an adaptive atmospheric intensity estimation method can effectively estimate atmospheric automatic light intensity, while using a fast dual filtering algorithm transmittance repair. By comparing the results of the analysis, the proposed algorithm can obtain good recovery image quality, while a substantial increase in processing speed, in order to obtain a more approximate image under sunny weather conditions, natural images, the paper further recovery image brightness adjustment, in order to achieve better visual effect.

\section{References}

[1]R.T.Tan. Visibility in bad weather from a single image[C],in Proc. IEEE Conf. Computer Vision and Pattern Recognition,1-8,2008.

[2]Srinivasa G. Narasimhan, Shree K. Nayar.Vision and the atmosphere[J].International Journal of Computer Vision ,2002,48(3):233-254.

[3]R. Fattal. Single image dehazing[J], in Proc. ACM SIGGRAPH,2008:1-9.

[4]Tarel J P, Hautiere N. Fast visibility restoration from a signal color or gray level image[C],in proc. IEEE Conf. Computer Vision,2009:2201-2208.

[5]He K M,Sun J ,Tang X O. Single image haze removal using dark channel prior[C] ,in Proc. IEEE Conf. Computer Visionand Pattern Recognition,2009:1956-1963.

[6]Hautiere N,Tarel J P ,Aubert D,et al. Blind contrast enhancement assessment by gradient ratioing at visible edges [J].Image Analysis and Stereology Journal,2008,27(2):87-95.

[7]Levin A, Lischinski D, Weiss $\mathrm{Y}$.A closed form solution to natural image matting [J].IEEE Transactions on Pattern Analysis and Machine Intelligence,2008,30(2):228-242.

[8]Yang Q X, Tan K H, Ahuja N. Real-time O(1) bilateral filtering[C]. in Proc. IEEE Conf. Computer Vision and Pattern Recognition. 2009:557-564.

[9]Paris S, Durand F.A fast approximation of the bilateral filter using a signal processing approach[J].International Journal of Computer Vision.2009,81(1):24-52. 